\title{
Financial Brownian Particle in the Layered Order-Book Fluid and Fluctuation-Dissipation Relations
}

\author{
Yoshihiro Yura, ${ }^{1,4}$ Hideki Takayasu, ${ }^{2,3}$ Didier Sornette, ${ }^{4}$ and Misako Takayasu ${ }^{1, *}$ \\ ${ }^{1}$ Department of Computational Intelligence and Systems Science, Interdisciplinary Graduate School of Science and Engineering, \\ Tokyo Institute of Technology 4259 Nagatsuta-cho, Yokohama 226-8502, Japan \\ ${ }^{2}$ Sony Computer Science Laboratories, 3-14-13, Higashi-Gotanda, Shinagawa-ku, Tokyo 141-0022, Japan \\ ${ }^{3}$ Meiji Institute for Advanced Study of Mathematical Sciences, Meiji University, \\ 4-21-1 Nakano, Nakano-ku, Tokyo 164-8525, Japan \\ ${ }^{4}$ Department of Management, Technology, and Economics, ETH Zurich, Scheuchzerstrasse 7, 8092 Zurich, Switzerland
}

(Received 12 April 2013; published 7 March 2014)

\begin{abstract}
We introduce a novel description of the dynamics of the order book of financial markets as that of an effective colloidal Brownian particle embedded in fluid particles. The analysis of comprehensive market data enables us to identify all motions of the fluid particles. Correlations between the motions of the Brownian particle and its surrounding fluid particles reflect specific layering interactions; in the inner layer the correlation is strong and with short memory, while in the outer layer it is weaker and with long memory. By interpreting and estimating the contribution from the outer layer as a drag resistance, we demonstrate the validity of the fluctuation-dissipation relation in this nonmaterial Brownian motion process.
\end{abstract}

DOI: 10.1103/PhysRevLett.112.098703

The mathematical description of fluctuation phenomena in statistical physics - of quantum fluctuation processes in elementary particle and field physics on the one hand, and of financial prices on the other-has a long tradition since Bachelier's seminal $1900 \mathrm{Ph} . \mathrm{D}$. thesis [1], which is anchored in the random walk model and the Wiener process. In physics, this field of study started from Einstein's 1905 paper on Brownian motion; the concept of the generalized Langevin equation and the fluctuationdissipation relations (FDRs) were well established in the middle of the 20th century [2,3]. Recently, strong interest has developed to clarify the conditions under which the FDRs are violated, based on numerical simulations [4,5] as well as experimental investigations using precise observations; the latter use advanced nanotechnology instruments for various materials such as colloidal suspension [6,7], polymers [8], liquid crystal [9], and glass systems [10].

The financial economic literature uses the Wiener process as the standard starting point for modeling and financial engineering applications [11]. Extending the initial intuition of Bachelier, the random nature of financial price fluctuations is presently mostly understood as resulting from the imbalance of buy and sell orders at each time step [12]. In order to explain non-Gaussian properties of market price fluctuations, extensions in the form of Langevin-type equations with an inertia term have been proposed [13-20].

Published by the American Physical Society under the terms of the Creative Commons Attribution 3.0 License. Further distribution of this work must maintain attribution to the author(s) and the published article's title, journal citation, and DOI.
PACS numbers: 89.65.Gh, 05.40.Jc, 82.70.Dd, 89.75.-k

Essentially all previous models based on the random walk picture or its continuous version (the Wiener process) involve just the price dynamics. Other approaches simulate financial markets by computational economic models with different classes of agent strategies, or by using the statistics of buy and sell orders from the viewpoint of statistical physics [21-28].

Here, we introduce a qualitatively novel type of model for financial price fluctuations. Rather than focusing on the dynamics of a single price for a given market that requires complicated modifications to the basic random walk model in order to account for the numerous stylized facts, we propose the picture that the observed financial motion is analogous to a genuine colloidal Brownian particle embedded in a fluid of smaller particles, which themselves reflect the structure of the underlying order book (defined as the time-stamped list of requests for buy and sell orders with prices and volumes). The "Financial Brownian Particle in order-book molecular matterantimatter fluid" (FBP, in short) picture provides a novel quantification of the correlations between different layers in the order book that can be interpreted as the analogy of the correlation between a Brownian particle itself with the surrounding fluid molecules. We present empirical estimations of the correlation functions that confirm the proposed mapping as well as provide nontrivial insights on the correlations with deeper fluid molecular layers within the order book.

We analyze the order-book data of the Electronic Broking Services for currency pairs provided by market managing company ICAP. This foreign exchange market is continuously open 24 hours per day, except over weekends, and its transaction volume per day, at about four trillion 
U.S. dollars, makes it the largest among all financial markets, with also much larger liquidity than stock markets. We present our results for the U.S. dollar-Japanese yen market, which is characterized by a large transaction volume. The traders in this market are international financial companies that are connected to ICAP's market server by a special computer network. Any orders, either buy or sell, are quantized by a unit of one million U.S. dollars with the price given with a granularity of 0.001 yen (called a pip) recorded with a time stamp of one millisecond. A pair of buy and sell orders meeting at the same price immediately triggers a transaction and determines the latest official market price. These orders disappear from the order book just like a pair annihilation of matter-antimatter. The price and time quantizations enable us to describe the market by particles in discrete space and time, where a particle represents either a buy or sell order of one million U.S. dollars. In the following discussion, we assign a superscript $-(+)$ for buy (sell) orders.

At a given time, a state of this market is characterized by its order book schematically represented in Fig. 1(a), which contains the set of yet unrealized buy (sell) orders in the lower (higher) side of the discrete price axis. The highest buy (lowest sell) order price, denoted as $x^{-}(t)\left(x^{+}(t)\right)$ is called the best bid (ask), and the gap between the best bid and best ask is called the spread. For each buy (sell) order in the order book, we introduce an important measure of depth $\gamma^{-}\left(\gamma^{+}\right)$, which is defined by the distance of this buy (sell) order from $x^{-}(t)\left(x^{+}(t)\right)$ in pip units.

The order book is evolved by the spontaneous injection of three types of orders: limit orders, market orders, and

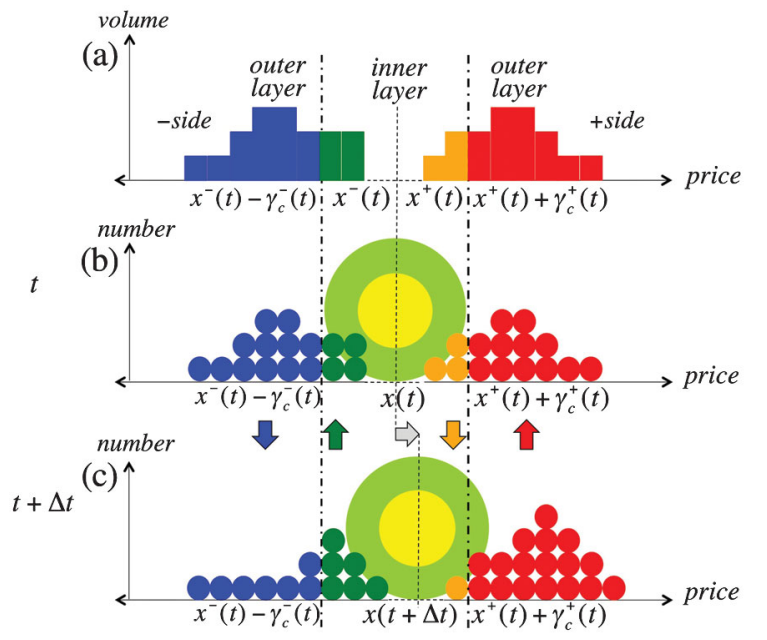

FIG. 1. Schematic representation of the FBP model. (a) An order-book configuration of buy orders (blue in outer layer and green in inner layer) and sell orders (red in outer layer and orange in inner layer) on the price axis. (b) Corresponding configuration of outer-layer particles (blue and red disks), inner-layer particles (green and orange disks), the colloidal Brownian particle's interaction range (yellow-green torus), and the core (yellow). (c) After $\Delta t$, the configuration of surrounding particles changes. cancellation. A limit buy (sell) order is introduced by a trader by specifying the buying (selling) price. If the buying (selling) price is lower than the best ask (higher than the best bid) in the order book, the order is accumulated at the specified price in the order book as a new buy (sell) order. If the buying (selling) price is equal to or higher than the best ask (lower than the best bid), this order makes a deal with a sell order at the best ask (bid) in the order book, and that pair of orders annihilates. A market buy (sell) order directly hits against a sell (buy) order at the best ask (bid), causing a deal. A cancellation simply deletes an order, and it can be done only by the trader who created the order. This highly irreversible particle dynamics evokes chemical catalysis, and leads to a rich phenomenology [29-35].

The FBP model that we propose is illustrated in Fig. 1(b). An imaginary colloidal Brownian particle, called a colloid, has its center positioned at the midprice, $x(t)=\left\{x^{-}(t)+\right.$ $\left.x^{+}(t)\right\} / 2$, with the core diameter given by the spread $x^{+}(t)-x^{-}(t)$. The accumulated orders are regarded as embedding fluid particles with a diameter equal to 1 pip. We visualize the core of the colloid by the yellow disk and the interaction range by the yellow-green ring area that overlaps with the particles near the spread (the green and orange disks). We call this interaction range the inner layer and the domain outside of this interaction range the outer layer. The values of threshold depths for defining the inner layer, $\gamma_{c}^{-}$and $\gamma_{c}^{+}$, will be estimated below from the data. With the injection of new orders, the surrounding particles change their configuration and the colloid moves as a result, as shown in Fig. 1(c) for a specific example. The colored arrows indicate typical particle density changes in the layers, which we are going to analyze in detail.

Observing the evolution of the configuration of particles from time $t$ to $t+\Delta t$, we measure the change in the number of $-(+)$ particles as a function of the depth $\gamma^{-}\left(\gamma^{+}\right)$, where the depth is measured from $x^{-}(t)\left(x^{+}(t)\right)$, at each time. When $x^{-}(t)\left(x^{+}(t)\right)$ stays at the same location, the change in particle number at a given depth is simply given by counting the change in the number of $-(+)$ particles. When $x^{-}(t)\left(x^{+}(t)\right)$ moves, the density profile as a function of depth shifts accordingly and the changes of particle numbers at different depth are simply due to the translation. Note that the depth $\gamma^{-}\left(\gamma^{+}\right)$can take a negative value, for example, in the case when a limit order falls in the spread.

Figure 2(a) shows the correlations between the change in the number of - (+) particles at each depth $\gamma, \Delta N_{\gamma}$, versus the velocity of the colloid, $v(t)=[x(t+\Delta t)-x(t)] / \Delta t$, where the time is in units of tick time, incremented by one unit when a deal occurs. In this figure, the value of the observation window is $\Delta t=100$. On average, this time interval corresponds to $160 \mathrm{sec}(1 \mathrm{tick} \simeq 1.6 \mathrm{sec})$. The velocity correlation with the change in the number of particles is negative for $\gamma^{-} \leq \gamma_{c}^{-}$, as shown by the orange line, and the correlation is positive for $\gamma_{c}^{-}<\gamma^{-}$, shown by the red line, where the estimated value of the boundary 

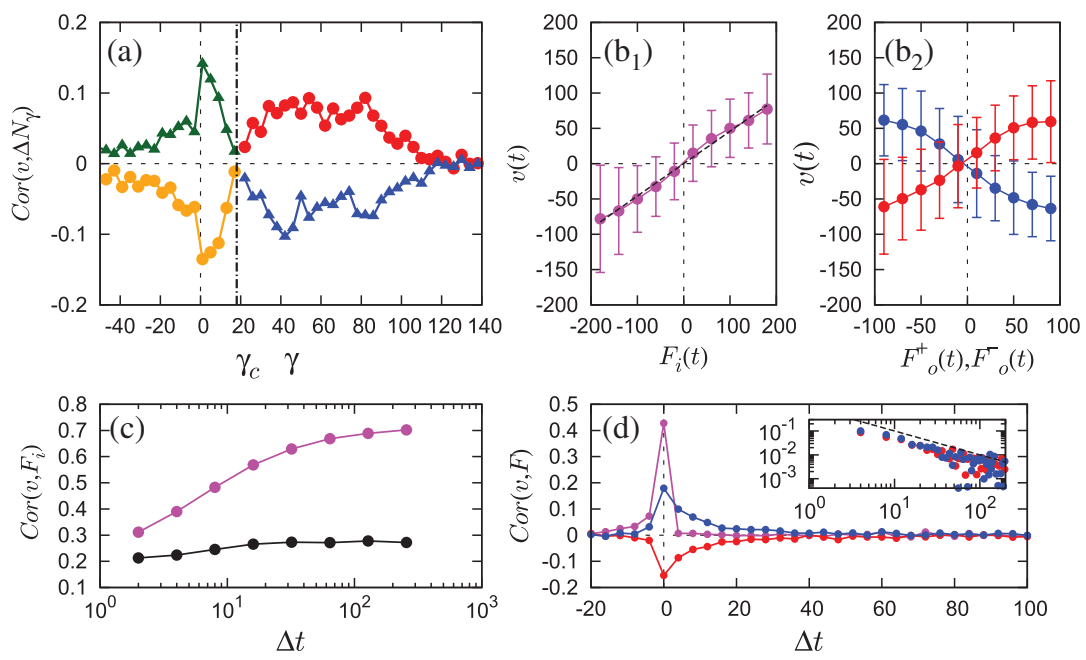

FIG. 2. Statistical properties supporting the physical Brownian analogy in Fig. 1. (a) Correlation function between velocity, $v(t)$, and the change in particle number at depth $\gamma$ for buy orders (green and blue triangles) and sell orders (orange and red circles) where $\gamma_{c}=18$. $\left(\mathrm{b}_{1}\right)$ Scatter plot of $v(t)$ as a function of $F_{i}(t)$ for $\Delta t=10^{2} .\left(\mathrm{b}_{2}\right)$ Scatter plot of $v(t)$ as a function of the change in the particle number in the outer layer for buy orders $\left[F_{o}^{-}(t)\right]$ and for sell orders $\left[F_{o}^{+}(t)\right]$ with $\Delta t=10^{2}$ (blue line and red line, respectively). Dots and bars represent the mean values and the standard deviations. (c) Correlation coefficients between $v(t)$ and $F_{i}(t)$ (pink dots), and between $v(t)$ and the "order flow" (black dots), defined by the number of buy orders minus that of sell orders. (d) Time-shifted correlation functions between $v(t)$ and $\left\{F_{i}(t+\Delta t), F_{o}^{+}(t+\Delta t), F_{o}^{-}(t+\Delta t)\right\}$ (pink line, blue line, and red line, respectively). The inserted figure shows a $\log -\log$ plot of these correlation functions as a function of $\Delta t$ and the dotted line shows a power law, $1 / \Delta t$.

is $\gamma_{c}^{-}=18$. The same relations with the opposite sign hold for + particles, as shown by the green and blue lines, implying that the dynamics is close to symmetric. Intuitively, when the price goes up, more new buy than sell orders are injected in the inner range and sell orders near the market price tend to be canceled and replaced by higher sell prices, based on the anticipation of larger future returns by traders assuming trend persistence. The opposite direction is explained in the same way.

Let us define the total change of particle numbers in the inner layer at time $t, f_{i}(t)=c_{i}^{-}(t)-c_{i}^{+}(t)-a_{i}^{-}(t)+$ $a_{i}^{+}(t)$ where $c_{i}^{-}(t)$ and $a_{i}^{-}(t)$ denote the numbers of particles that are created and annihilated, respectively, in the inner layer at time $t$, and $c_{i}^{+}(t)$ and $a_{i}^{+}(t)$ are the same quantities for + particles. Note that - and + particles are counted with the opposite sign as they are conjugate "matter" and "antimatter." In Fig. 2 (b $\left.b_{1}\right)$, the scatter plot of the velocity of the colloid, $v(t)$, observed in the same time window, $\Delta t=100$, as a function of the $\operatorname{sum} F_{i}(t)=$ $\sum_{s=0}^{\Delta t} f_{i}(t+s)$ from $t$ to $t+\Delta t$ demonstrates a strong linear correlation. Figure 2(c) shows the correlation coefficient between $v(t)$ and $F_{i}(t)$ for different values of $\Delta t$. The correlation increases for larger $\Delta t$, reaching the value 0.7 around $\Delta t=100$. These empirical results suggest the following basic relation:

$$
v(t)=L(\Delta t)(1 / \Delta t) \sum_{s=0}^{\Delta t} f_{i}(t+s)+\eta(t) .
$$

The factor $L(\Delta t)$ represents the mean step length of the colloid motion as a response to the motion of the surrounding fluid particles. It ranges from $L(\Delta t) \approx 0.44$ pips for $\Delta t=100$ to $L(\Delta t) \approx 0.32$ pips for $\Delta t=4$. The last term, $\eta(t)$, is the error term. Similar correlations between the velocity and market orders have been previously documented [29]. However, we find here significantly higher values due to the more appropriate separation of the negative and positive sides of the layered structure of the order book. We also observed, similarly, the changes in outer-layer particle numbers for buy orders and sell orders separately, and confirmed the correlations with the velocity as shown in Fig. 2(b $\left.b_{2}\right)$. Figure 2(d) shows the time-shifted correlations between the velocity and these changes in particle numbers, which confirms that the inner-layer particles correlate strongly with the velocity but with a short memory, while the correlation of the outer-layer particles is weaker but decays slowly with a power tail.

Next, we present a more detailed description of the fluid particles. Figure 3(a) shows a schematic diagram of the space-time configuration. We categorize the particles that annihilate in the inner layer into two classes, $a_{i i}$ and $a_{o i}$. An $a_{i i}$ particle is created in the inner layer, stays in the inner layer, and annihilates in the inner layer, while an $a_{o i}$ particle is born either in the inner layer or outer layer, visits the outer layer at least once, and then annihilates in the inner layer. By surveying the whole life of the particle, we find that $73.5 \%$ of particles are created in the inner layer (denoted as $c_{i}$ ), and $72.3 \%$ of particles are annihilated in the inner layer (denoted as $a_{i}$ ). The share of $a_{i i}$ particles is $61.5 \%$ and that of $a_{o i}$ is $10.8 \%$. 


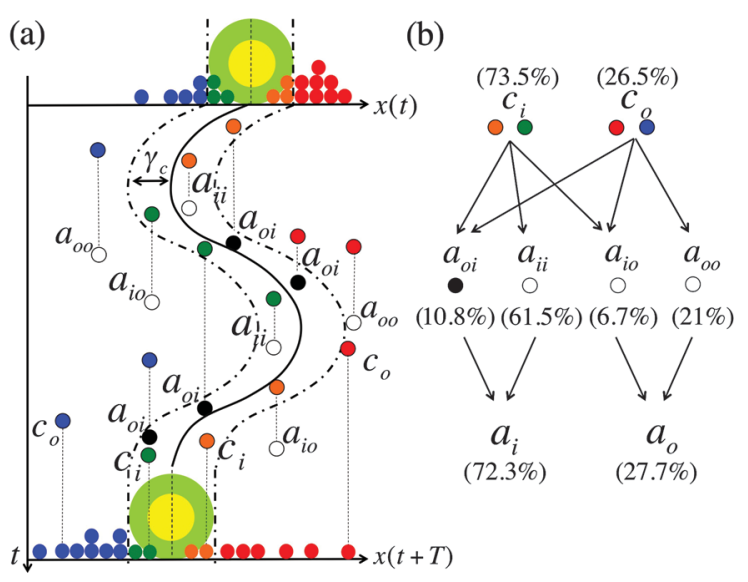

FIG. 3. Schematic diagram of the space-time configuration of particles. (a) Creation and annihilation of fluid particles are shown together with the trajectory of the colloid motion (black line) and the inner layer (the region between black dotted lines). The black disks represent $a_{o i}$ particles. (b) Share percentages of the different particle types.

Statistics of $a_{i i}$ and $a_{o i}$ are compared in Fig. 4. The timeshifted correlations with $v(t)$ are plotted in Fig. 4(a) for $g_{i i}(t)=-a_{i i}^{-}(t)+a_{i i}^{+}(t)$ by blue line and for $g_{o i}(t)=$ $-a_{o i}^{-}(t)+a_{o i}^{+}(t)$ by red line. We find that $a_{i i}$ and $a_{o i}$ are oppositely correlated with the velocity. Figure 4(b) shows
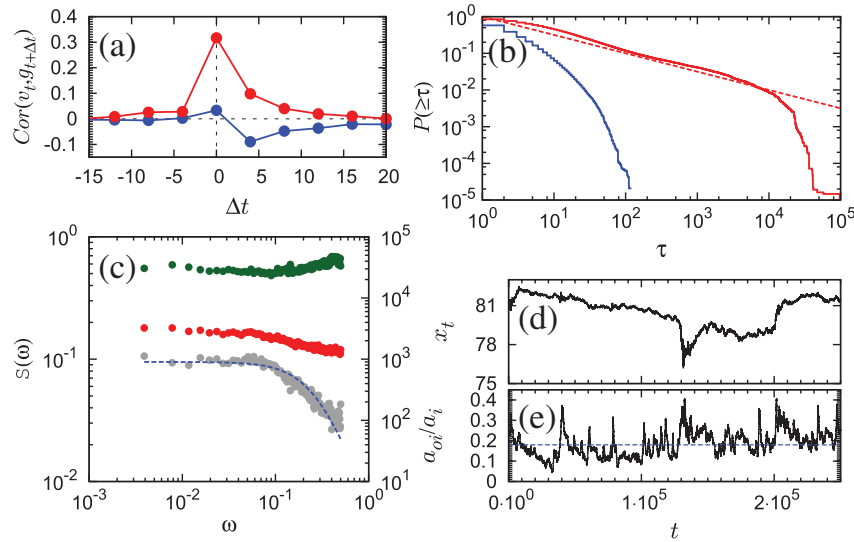

FIG. 4. Statistical properties of $a_{o i}$ particles (see text for definition): (a) Correlation functions of $\left\{v(t), g_{o i}(t+\Delta t)\right\}$ (upper red) and $\left\{v(t), g_{i i}(t+\Delta t)\right\}$ (lower blue). (b) Cumulative lifetime distribution of $a_{i i}$ (left blue) and $a_{o i}$ (right red), with the power law $\tau^{-0.5}$ shown as a guideline (red dotted line). (c) Power spectra of $v(t) \Delta t$ (top green), $v_{I}(t) \Delta t$ (middle red), and ratio of $\left|v_{I}^{2}(\omega)\right| /\left|v^{2}(\omega)\right|$ (bottom gray), with the blue dotted guideline $a /\left(b+\omega^{2}\right)$ with $(\mathrm{a}, \mathrm{b})=(0.00725,0.0764)$. The power spectra are averaged over 200 samples of time series, of size $2^{8}$ ticks. The right vertical axis is for $v(t) \Delta t$ and $v_{I}(t) \Delta t$, and the left vertical axis is for the ratio. (d) Time series of $x(t)$ (U.S. dollarsJapanese yen exchange rates for the week of March 14, 2011). (e) Time series of the ratio $a_{o i} / a_{i}$, measured for the time interval $[t-1000, t]$, and the averaged value for the whole period (the blue dotted line). the cumulative distributions of the lifetimes of these particles in log-log scale. The distribution of the lifetimes of $a_{i i}$ particles decays exponentially with a mean lifetime of approximately 2.6 ticks, while that of $a_{o i}$ follows a power law with an exponent close to -0.5 , which corresponds to the distribution of recurrence time intervals for one-dimensional random walks.

These results justify a more sophisticated FBP picture in which the $a_{i i}$ particles contribute to the driving force, directly pushing or pulling the colloid at the time of annihilation; the $a_{o i}$ particles work as a drag that impede the colloidal motion since they always collide with the front of the colloidal motion. Based on this picture, the velocity equation, Eq. (1), is decomposed as $v(t)=$ $v_{I}(t)+v_{F}(t)+\eta(t)$, where $v_{I}(t)=L(\Delta t) \sum_{s=0}^{\Delta t}\left\{-a_{o i}^{-}(t+\right.$ $\left.s)+a_{o i}^{+}(t+s)\right\} / \Delta t$ and $v_{F}(t)=L(\Delta t) \sum_{s=0}^{\Delta t}\left\{c_{i}^{-}(t+s)-\right.$ $\left.c_{i}^{+}(t+s)-a_{i i}^{-}(t+s)+a_{i i}^{+}(t+s)\right\} / \Delta t$.

As the term $v_{F}(t)$ is nothing but the direct driving force term that reflects the immediate orders of traders, we focus on the term $v_{I}(t)$, which is caused by the long-term response of the fluid particles. The power spectra of $v(t) \Delta t, v_{I}(t) \Delta t$, and their ratio are plotted in Fig. 4(c). The power spectrum of $v(t)$ is nearly white with slightly more energy in the high frequency band, implying that there are zigzag fluctuations at very short times. On the other hand, the spectrum of $v_{I}(t) \Delta t$ clearly decays at high frequency. The ratio of power spectra, $\left|v_{I}(\omega)\right|^{2} /|v(\omega)|^{2}$, has a Lorentzian form, implying that the response function is approximated by an exponential function $v_{I}(t)=$ $\int_{-\infty}^{t} \phi(t-s) v(s) d s, \phi(t)=\phi_{0} e^{-\delta t}$ with the following estimated parameter values: $\phi_{0}=0.08$ and $\delta=0.27$. Introducing these relations into $v(t)=v_{I}(t)+v_{F}(t)+\eta(t)$ and taking its time derivative, we obtain the following standard form of a Langevin equation in the continuous time representation, which demonstrates the validity of the FDR:

$$
(d / d t) v(t)=-\mu v(t)+G(t), \quad \mu=\left(\delta / \phi_{0}\right)-1 .
$$

The external force term $G(t)$ includes $v_{F}, \eta$, and their derivatives, which are not simple white noise, and the drag coefficient is estimated as $\mu=2.2$. The validity of this continuum formulation can be checked by estimating the Knudsen number [36-38] of the financial markets, defined as the ratio of the mean free path of collisions of the colloid and the $a_{o i}$ particles over the diameter of the colloid. We find that the averaged value of the Knudsen number is approximately 0.02 , the smallness of which guarantees the validity of the continuum representation of market price given by Eq. (2).

So far, we have analyzed the whole data set to observe the averaged behaviors, neglecting the well-known fact that markets are not stationary and are characterized by regime shifts: Calm periods are punctuated by turbulent periods of high transient volatility, including speculative bubbles and 
crashes. It is thus more appropriate to revisit our above estimations of observables in shorter time scales and analyze their possible time variation. A detailed analysis will be described in a separate paper. Here, we show the temporal change of the ratio of $a_{o i} / a_{i}$ in Fig. 4(e) with the corresponding market price in Fig. 4(d). In addition to being easy to observe, the ratio $a_{o i} / a_{i}$ constitutes the key parameter related to the strength of the drag force exerted by the fluid particles. One can see that $a_{o i} / a_{i}$ fluctuates significantly, confirming that market conditions are not stationary.

In summary, we have established a fundamental analogy between the motion of a colloidal particle embedded in a fluid and the price dynamics of a financial market in the order book. By observing the detailed behaviors of the colloid and surrounding particles in the order book, we found that the drag resistance is caused by particles moving from the outer layer to the inner layer. The proposed quantitative correspondence provides a novel perspective for the analysis of financial markets. In addition, it should provide a stimulus for physicists from many different fields, since the question of the origin of drag resistance is a fundamental question in physics that still remains to be fully clarified. We also showed the need to enhance the analysis by accounting for the nonstationary properties of markets. Moreover, there are some market conditions where we find that the Knudsen number becomes close to 1 , requiring the extension of the continuous description (2) into a discrete representation. Our approach demonstrates the importance of physical intuition associated with financial insights in analyzing the big data of financial markets.

The authors appreciate useful comments by Professor Hisao Hayakawa. This work was partially supported by Grant-in-Aid for Scientific Research (C) No. 24540395. Y. Y. was financially supported by Japan Society for the Promotion of Science, Research Fellowship for Young Scientists.

*takayasu@dis.titech.ac.jp

[1] L. Bachelier, Annales Scientifiques de l'École Normale Supérieure 17, 21 (1900).

[2] R. Kubo, Rep. Prog. Phys. 29, 255 (1966).

[3] H. Mori, Prog. Theor. Phys. 33, 423 (1965).

[4] F. Sciortino and P. Tartaglia, Phys. Rev. Lett. 86, 107 (2001).

[5] H. Kawamura, Phys. Rev. Lett. 90, 237201 (2003).

[6] C. Maggi, R. Di Leonardo, J. C. Dyre, and G. Ruocco, Phys. Rev. B 81, 104201 (2010).

[7] D. R. Strachan, G. C. Kalur, and S. R. Raghavan, Phys. Rev. E 73, 041509 (2006).
[8] H. Oucris and N. E. Israeloff, Nat. Phys. 6, 135 (2009).

[9] S. Joubaud, B. Percier, A. Petrosyan, and S. Ciliberto, Phys. Rev. Lett. 102, 130601 (2009).

[10] K. Komatsu, D. L'Hôte, S. Nakamae, V. Mosser, M. Konczykowski, E. Dubois, V. Dupuis, and R. Perzynski, Phys. Rev. Lett. 106, 150603 (2011).

[11] R. C. Merton, Bell Journal of economics 4, 141 (1973).

[12] A. S. Kyle, Econometrica 53, 1315 (1985).

[13] T. Hirabayashi, H. Takayasu, H. Miura, and K. Hamada, Fractals 01, 29 (1993).

[14] J. D. Farmer, Ind. Corp. Change. 11, 895 (2002).

[15] J.-P. Bouchaud and R. Cont, Eur. Phys. J. B 6, 543 (1998).

[16] K. Ide and D. Sornette, Physica (Amsterdam) 307A, 63 (2002).

[17] H. Takayasu, A.-H. Sato, and M. Takayasu, Phys. Rev. Lett. 79, 966 (1997).

[18] M. Takayasu, T. Mizuno, and H. Takayasu, Physica (Amsterdam) 370A, 91 (2006).

[19] K. Watanabe, H. Takayasu, and M. Takayasu, Phys. Rev. E 80, 056110 (2009).

[20] Y. Yura, H. Takayasu, K. Nakamura, and M. Takayasu, J. Stat. Comp. Simu. (to be published).

[21] S. V. Vikram and S. Sinha, Phys. Rev. E 83, 016101 (2011).

[22] P. Bak, M. Paczuski, and M. Shubik, Physica (Amsterdam) 246A, 430 (1997).

[23] D. L. C. Chan, D. Eliezer, and I. I. Kogan, arXiv:cond-mat/ 0101474

[24] D. Challet and R. Stinchcombe, Physica (Amsterdam) 300A, 285 (2001).

[25] F. Slanina, Phys. Rev. E 64, 056136 (2001).

[26] V.Plerou, P. Gopikrishnan, and H. E. Stanley, Phys. Rev. E71, 046131 (2005).

[27] H. Takayasu, H. Miura, T. Hirabayashi, and K. Hamada, Physica (Amsterdam) 184A, 127 (1992).

[28] K. Yamada, H. Takayasu, T. Ito, and M. Takayasu, Phys. Rev. E 79, 051120 (2009).

[29] J. Hasbrouck, Empirical Market Microstructure: The Institutions, Economics and Econometrics of Securities Trading (Oxford University Press, New York, 2007).

[30] S. Maslov, Physica (Amsterdam) 278A, 571 (2000).

[31] J.-P. Bouchaud, Y. Gefen, M. Potters, and M. Wyart, Quant. Finance 4, 176 (2004).

[32] J. D. Farmer, A. Gerig, F. Lillo, and S. Mike, Quant. Finance 6, 107 (2006).

[33] F. Lillo, J. D. Farmer, and R. N. Mantegna, Nature (London) 421, 129 (2003).

[34] P. Weber and B. Rosenow, Quant. Finance 5, 357 (2005).

[35] R. Cont, A. Kukanov, and S. Stoikov, J. Financ. Econ. 12, 47 (2013).

[36] M. Knudsen, Ann. Phys. (Leipzig) 28, 75 (1909).

[37] E. Cussler, Diffusion: Mass Transfer in Fluid Systems, Cambridge Series in Chemical Engineering (Cambridge University Press, Cambridge, England, 1997) 3rd ed.

[38] J. T. Padding and A. A. Louis, Phys. Rev. E 74, 031402 (2006). 